\title{
PERIODIC ELECTRON MOTION IN THE FIELD OF INTENSIVE PLANE LIGHT WAVE
}

\author{
Yu.N. Grigor'ev*, O.D. Zvonaryova \\ National Science Center "Kharkov Institute of Physics\&Technology", Kharkov, Ukraine
}

\section{Abstract}

Results of integration of the Lorentz-Dirac equation for electron motion in the field of intensive light wave are given in this work. Diagrams and parameters, which characterise the periodic motion of a relativistic electron, are shown. Formulas of the spectral-angular distribution of the electromagnetic field irradiated by a relativistic electron, moving towards electromagnetic wave, were obtained.

\section{INTRODUCTION}

In [1] the Dirac-Lorentz equation for motion of a relativistic electron in the field of travelling linearly polarized plane light wave was integrated. The bremsstrahlung force produced by the electron radiation was taking into account. It was shown, that the bremsstrahlung force results in an appearance of decrement. However, under some conditions the periodic motion of a radiating electron is possible.

In the present work approximate conditions of the existence of periodic motion of a radiating electron are analyzed. Diagrams of electron periodic motion were calculated without taking into account the radiation. Formulas of spectral-angular distribution of electromagnetic radiation intensity for an electron, which is moving periodically in a wave field, were obtained.

\section{MOTION EQUATION}

The Dirac-Lorentz equation of electron motion in the electromagnetic theory [2] can be written as

$$
\frac{d}{d t} m \mathbf{v}=\mathbf{F}_{L}+\mathbf{F}_{R}
$$

$m=m_{0} /\left(1-\beta^{2}\right)^{\frac{1}{2}}, \beta=\frac{\mathrm{v}}{c}, m_{0}$ is the rest mass of an electron, $c$ is the light velocity, $\mathbf{v}$ is the vector of electron velocity, $\mathrm{F}_{L}$ is the Lorentz force, $\mathbf{F}_{R}$ is the bremsstrahlung force.

It could be shown [1] that when $\mathbf{F}_{R}=0$ in the field of plane electromagnetic wave the integral of motion takes place:

$$
\left(1+\beta_{x}(t)\right) /\left(1-\beta^{2}(t)\right)^{1 / 2}=\left(1+\beta_{x}(0)\right) /\left(1-\beta^{2}(0)\right)^{1 / 2}=B
$$

where $\beta_{x}=\left|\mathrm{v}_{x}\right| / c, \mathrm{v}_{\mathrm{x}}$ is the velocity projection onto the $\mathrm{x}$-axes, " 0 " designates a value at the initial time instant.

* Zelinsky@kipt.kharkov.ua

$$
\begin{aligned}
& \frac{d^{2} \mathbf{r}}{d S^{2}}=\frac{e}{\mu_{0}} \mathbf{E}+\frac{e}{\mu_{0} c} \mathbf{n}\left(\mathbf{E} \frac{d \mathbf{r}}{d S}\right)+ \\
& +\frac{2}{3} \frac{1}{\mu_{0}} r_{e}^{2} E_{a}^{2} \cos ^{2}(2 \pi v S+\delta)\left[-\mathbf{i}-\frac{\left(1+\beta_{x}(0)\right)^{2}}{\left(1-\beta^{2}(0)\right)} \frac{d \mathbf{r}}{d S} / c\right]+ \\
& +\frac{2}{3} \frac{1}{\mu_{0}} \frac{2 \pi r_{e} e E_{a} \sin (2 \pi v S+\delta)}{\lambda} \frac{\left(1+\beta_{x}(0)\right)}{\sqrt{1-\beta^{2}(0)}} \times \\
& \times\left[-\mathbf{k}-\mathbf{i} \frac{d z}{d S} / c\right],
\end{aligned}
$$

where $r_{e}=e^{2} / m_{0} c^{2}$ is the radius of electron, $\lambda=c / v$ is the light wavelength, $\mu_{0}=m_{0} B, \mathbf{k}$ and $\mathbf{i}$ are unit vectors of the z-axes and $\mathrm{x}$-axes, respectively.

$$
E_{z}=E_{a} \cos \left[2 \pi v\left(t-\frac{\mathbf{n} \mathbf{r}}{c}\right)+\delta\right], \mathbf{H}=[\mathbf{n E}],
$$

where $\mathbf{n}$ is the normal to the wave front, $\mathbf{r}$ is a radiusvector, $\delta$ is the phase initial value. $\mathbf{E}, \mathbf{H}$ are vectors of electric and magnetic fields, respectively.

$$
S=t-\frac{\mathbf{r n}}{c} ; \frac{d S}{d t}=1-\beta_{x} ; \frac{d S}{d t}=B \sqrt{1-\beta^{2}}
$$

\section{PERIODIC SOLUTION}

It could be shown that Eq. (3) under some conditions has a periodic solution. Approximate conditions for electron periodic motion without taking into account bremsstrahlung force $\left(\mathbf{F}_{R} \equiv 0\right)$ are reduced to following expressions

$$
\begin{aligned}
& y^{\prime}(0)=0 ; z^{\prime}(0)=p \sin \delta ; p=\frac{e E_{a}}{\mu_{0}(2 \pi v)} \\
& \int_{0}^{v^{-1}} \sqrt{L \cos ^{2} 2(2 \pi \nu S+\delta)+M \cos 2(2 \pi \nu S+\delta)+N} d \xi= \\
& B v^{-1}\left[1+a_{1} / c\right] \\
& L=2^{-4} c^{-4} B^{2} p^{4} ; M=-\frac{1}{2} p^{2} c^{-2} B^{2}\left[a_{1} / c+1\right] \\
& N=\left(a_{1}^{2}+\frac{p^{2}}{2}\right) c^{-2} B^{2}+1 \\
& a_{1}=x^{\prime}(0)+\frac{1}{4} \frac{p^{2}}{c} \cos 2 \delta \\
& z^{\prime}(0)=\frac{d z}{d S}=\frac{\beta c \sin \alpha}{1-\beta \cos \alpha} ; x^{\prime}(0)=\frac{d x}{d S}=\frac{\beta c \cos \alpha}{1-\beta \cos \alpha}
\end{aligned}
$$

where $\alpha$ is the angle between initial directions of $\mathbf{v}$ and $\mathbf{i}$. 
Eq.(6) is the condition of electron periodic motion with the period $v^{-1}$ on z. Eq.(7) is the condition of periodicity on time. It was obtained from the requirement that, when electron passed along $\mathrm{x}$-axis the distance equal to $a_{1} v^{-1}$, it came to the same wave phase. The numerical calculations have shown, that Eq.(7) is the result of Eq.(6). To obtain the spectral - angular distribution of radiation intensity of electron moving in the $\mathrm{xz}$ plane, we calculate electric field components in the wave zone [2]:

$$
\begin{aligned}
& \boldsymbol{E}_{x}(l w)=\frac{l e^{i w R / c}}{c^{2} R} \frac{(-i l w)}{T} \int_{0}^{v^{-1}}\left(-x^{\prime} \sin ^{2} \varphi+z^{\prime} \sin \varphi\right) e^{i k w F(s)} d S \\
& \boldsymbol{E}_{z}(l w)=\frac{l e^{i w R / c}}{c^{2} R} \frac{(-i l w)}{T} \int_{0}^{v^{-1}}\left(-z^{\prime} \cos ^{2} \varphi+x^{\prime} \cos \varphi\right) e^{i l w F(S)} d S \\
& \boldsymbol{E}_{y}(l w)=0
\end{aligned}
$$

$\mathcal{E}_{x}, \mathcal{E}_{z}, \boldsymbol{E}_{y}$ are the projections of a radiating vector of electrical component.

$$
\begin{aligned}
& F(S)=S+\frac{x}{c}-\frac{1}{c}(x \cos \varphi+z \sin \varphi) \\
& x^{\prime}=\frac{p^{2}}{4 c} \cos 2(2 \pi v S+\delta)+a_{1} ; z^{\prime}=p \sin (2 \pi v S+\delta) \\
& x=-\frac{p^{2}}{4 c(4 \pi v)} \sin 2(2 \pi v S+\delta)+a_{1} S+a_{2} \\
& z=-\frac{p}{(2 \pi v)} \cos (2 \pi v S+\delta)+c_{2} \\
& a_{2}=x(0)+\frac{p^{2}}{4 c(4 \pi v)} \sin 2 \delta ; c_{2}=z(0)+\frac{p}{2 \pi v} \cos \delta
\end{aligned}
$$

$\mathrm{R}$ is the distance from an electron to the point of observation, $\varphi$ is the angle between unit vector of $\mathrm{x}$-axis $\mathbf{i}$ and vector, directed from the coordinate origin to the point of observation. $T=v^{-1}\left[1+\frac{a_{1}}{c} / c\right]$ is the period of electron motion on the trajectory, $w=2 \pi / T$.

Using the given formulas, we can obtain fundamental frequencies of the electromagnetic field radiation in the point of observation:

$$
\begin{aligned}
& f_{1}=v /\left[1+(1-\cos \varphi) \frac{a_{1}}{c}\right] ; f_{2}=2 f_{1} \\
& l=\left(1+\frac{a_{1}}{c}\right) /\left[1+\frac{a_{1}}{c}(1-\cos \varphi)\right]
\end{aligned}
$$

$l$ is the number of radiation harmonic.

The formula for $l$ (whole number) puts a requirement on a relation between parameters of wave and particle, and ensures a linear spectrum of radiation, typical under a periodic electron motion on time.

During the calculation Eq.(12) the small oscillations $x$ and $\mathrm{z}$ were not taken into account. Small $\mathrm{x}$ and $\mathrm{z}$ will give higher radiation harmonics but very small in comparison with fundamental harmonics of radiation.

The known formula of maximum frequency of Compton scattering for $\boldsymbol{\varepsilon}_{\mathrm{z}}$ follows from Eq.(12):

$$
f_{1} \approx 4 \gamma^{2} v
$$

when $\alpha=\pi, \varphi=\pi, \gamma=W / m_{0} c^{2}, W$ is the electron energy.

The formula (13) is obtained at limitation on intensity of a plane wave $E_{a}$ :

$$
\frac{p}{c}=\left(\frac{e E_{a}}{\mu_{0}(2 \pi v)}\right) \ll<\frac{\gamma^{-1}}{\sqrt{2}}
$$

For example, at $\mathrm{W}=50 \mathrm{MeV}, v=3 * 10^{14} \mathrm{~Hz} E_{a}$ should be less than magnitude $\pi \sqrt{2} * 10^{8} \mathrm{~V} / \mathrm{m}$.

From these formulas the important result follows: with increasing of the incident wave intensity $E_{a}$ the frequency of electron radiation decreases and tends to frequency of the incident wave at very large values. It is connected with decreasing of velocity of electron, which oscillates in a wave field towards the observer. So, at $a_{l}=0$ the electron stops to move along the $\mathrm{x}$-axis and Doppler effect disappears. The electron reradiates the incident wave.

Figs. 1-3 show the trajectories of an electron with energy $50 \mathrm{MeV}$ in the field of a travelling wave for typical cases: $v=3^{*} 10^{14} \mathrm{~Hz}, \varphi=\pi$. 1) $\mathrm{Ea}=10^{8} \mathrm{~V} / \mathrm{m}, \alpha=\pi$ $\left.3^{*} 10^{-7}, \delta=1,3 ; \mathrm{f}_{1}=1,2 * 10^{19} \mathrm{~Hz} ; 2\right) \mathrm{Ea}=5^{*} 10^{14} \mathrm{~V} / \mathrm{m}, \alpha=\pi$ $\left.10^{-2}, \delta=0,0064, \mathrm{f}_{1}=9,93 * 10^{14} \mathrm{~Hz} ; 3\right) \mathrm{Ea}=9,1 * 10^{14} \mathrm{~V} / \mathrm{m}$, $\alpha=\pi-10^{-2}, \delta=0,0035, \mathrm{f}_{1}=3 * 10^{14} \mathrm{~Hz}$.

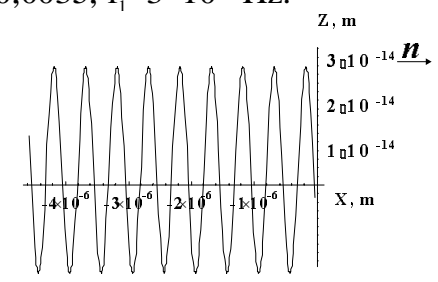

Figure 1

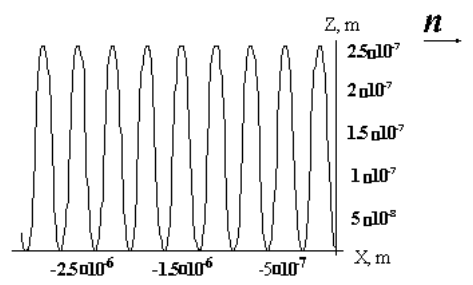

Figure 2

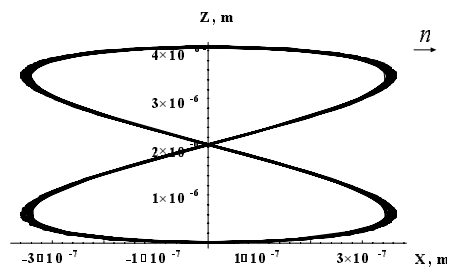

Figure 3

\section{REFERENCES}

[1] Yu.N. Grigor'ev, O.D. Zvonaryova, I.M. Karnaukhov. Electron motion in the field of intensive plane light wave, VANT, 2000, N2, p. 72-75.

[2] L.D. Landau and E.M. Lifshits A field theory. 1960, Nauka, Moscow, p. 416. 